\title{
Disseminated Histiocytic Sarcoma with Ureteric Involvement in a Jindo Dog
}

\section{Hee-Soo Cho' \\ Jong-Tae Cheong ${ }^{2}$ \\ Hyun-Jung Park ${ }^{3}$ \\ Jae-Hoon $\mathrm{Kim}^{4}$ \\ Ji-Youl Jung ${ }^{4}$ \\ Youngmin Yun ${ }^{1 * *}$ \\ Woo-Jin Song ${ }^{1, *}$}

'Laboratory of Veterinary Internal Medicine, College of Veterinary Medicine, Jeju National University, Jeju 63243, Korea

${ }^{2}$ Laboratory of Veterinary Surgery, College of Veterinary Medicine, Jeju National University, Jeju 63243, Korea

${ }^{3}$ Laboratory of Veterinary Imaging, College of Veterinary Medicine, Jeju National University, Jeju 63243, Korea

${ }^{4}$ Laboratory of Veterinary Pathology, College of Veterinary Medicine, Jeju National University, Jeju 63243, Korea

*Correspondence: ssong@jejunu.ac.kr (WooJin Song), dvmyun@jejunu.ac.kr (Youngmin Yun)

\section{ORCID}

Hee-Soo Cho:

https://orcid.org/0000-0003-0328-163X

Jong-Tae Cheong:

https://orcid.org/0000-0003-4141-718X

Hyun-Jung Park:

https://orcid.org/0000-0001-8856-5759 Jae-Hoon Kim:

https://orcid.org/0000-0002-4410-9126 Ji-Youl Jung:

https://orcid.org/0000-0002-5938-2029

Youngmin Yun:

https://orcid.org/0000-0003-0695-0843

Woo-Jin Song:

https://orcid.org/0000-0002-9195-551X

Copyright $\odot$ The Korean Society of Veterinary Clinics
Abstract We describe the case of a Jindo dog that presented with a 2-month history of an abscess-like mass on the skin of the left hip. The patient was initially diagnosed with a suspected insect bite and was treated with a topical dressing. After observing no improvements, surgical debridement was done to remove the abscess. Nine days after surgery, the patient started showing severe anorexia, vomiting, lethargy, and oliguria. Azotemia was detected, and the patient was diagnosed with acute kidney injury. Ultrasonography depicted an abdominal mass (4.6 $\times 7.5 \mathrm{~cm}$ ) in the right ureter, and severe hydronephrosis in the right kidney. The patient was euthanatized. Results of the necropsy, histopathologic examination, and immunohistochemistry for tumor cells, confirmed that the patient had developed hydronephrosis and disseminated histiocytic sarcoma which had metastasized to the lungs. To the best of our knowledge, this is the first report of acute renal failure produced by ureter invasion and urinary tract obstruction resulting from the abdominal mass of disseminated histiocytic sarcoma in dogs.

Key words acute renal failure, aminoglycosides, dog, disseminated histiocytic sarcoma, urinary tract obstruction. 


\section{Introduction}

Histiocytic sarcoma (HS) arises from the myeloid histiocytic lineage of Langerhans cells, interstitial dendritic cells, and macrophages (5). Canine HS can occur in a localized or disseminated form which is an aggressive multisystem disease characterized by the presence of multiple tumor masses in several organs $(1,7)$. Disseminated HS is most often found in the spleen, peripheral lymph nodes, liver, bone marrow, lungs, subcutis, skeletal muscles, and kidneys $(1,3,4)$. It has a potential for highly aggressive behavior; therefore, complete staging to determine the extent of the disease is recommended prior to therapy (5). It is a rapidly progressive disease with a poor prognosis, even with aggressive therapy. Currently, systemic chemotherapy using a variety of chemotherapeutic drugs is recommended.

Previous literature has reported responses to chemotherapy with Lomustine (CCNU) in dogs; an overall response rate of $44 \%$ was observed, including 5 complete remissions and 21 partial remissions. The median survival time of the responders was 172 days, and the overall median survival time for 59 dogs was 105 days (9). Studies have shown that thrombocytopenia, hypoalbuminemia, anemia, disseminated disease, and a lack of antitumor treatment were negative prognostic factors $(2,9)$. In addition, Dervisis et al. (2) reported that the concurrent use of corticosteroids appeared to increase the risk of death. This is the first report of acute renal failure produced by ureter invasion and urinary tract obstruction resulting from the abdominal mass of disseminated histiocytic sarcoma in a Jindo dog.

\section{Case Report}

An 8-year-old neutered, male Jindo dog presented with a 2-month history of an abscess-like mass $(4 \times 20 \mathrm{~cm})$ on the skin of the left hip (Fig. 1). The dog was treated with a topical dressing for a suspected insect bite, but after 2 months of no improvement, he was referred to the veterinary medical teaching hospital of College of Veterinary Medicine in Jeju National University. On physical examination, his weight was $32 \mathrm{~kg}$, and vital signs including systemic blood pressure, temperature, pulse, and respiratory rate, were normal. A complete blood count revealed leukocytosis (white cell count, $24.12 \times 10^{9} / \mathrm{L}$; reference range, $\left.5-12 \times 10^{9} / \mathrm{L}\right)$ without anemia. C-reactive protein levels were also elevated ( 24.8 $\mathrm{mg} / \mathrm{dL}$; reference range, $0-20 \mathrm{mg} / \mathrm{dL}$ ), and serum biochemical analysis results were unremarkable. Surgical debridement was performed to remove the abscess, and the wound site
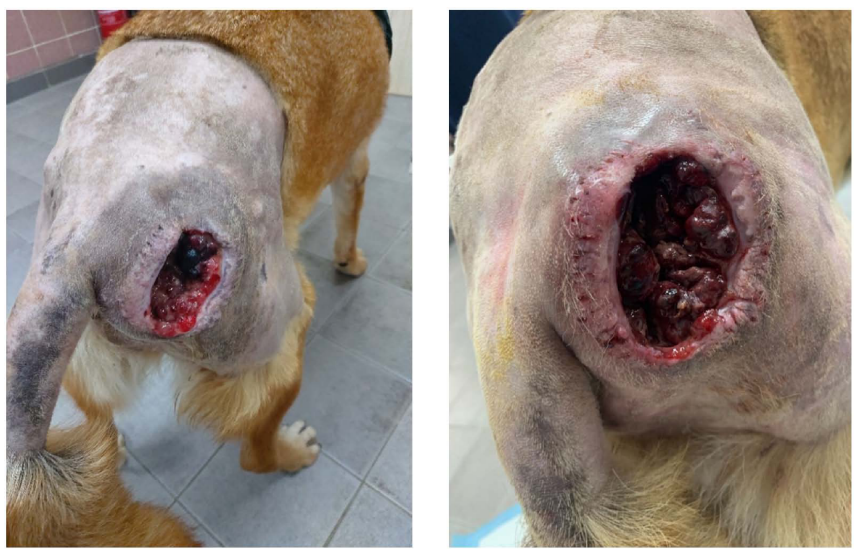

Fig. 1. A skin mass $(4 \times 20 \mathrm{~cm})$ with abscess on the left hip. There was no improvement with dressing or surgical debridement.
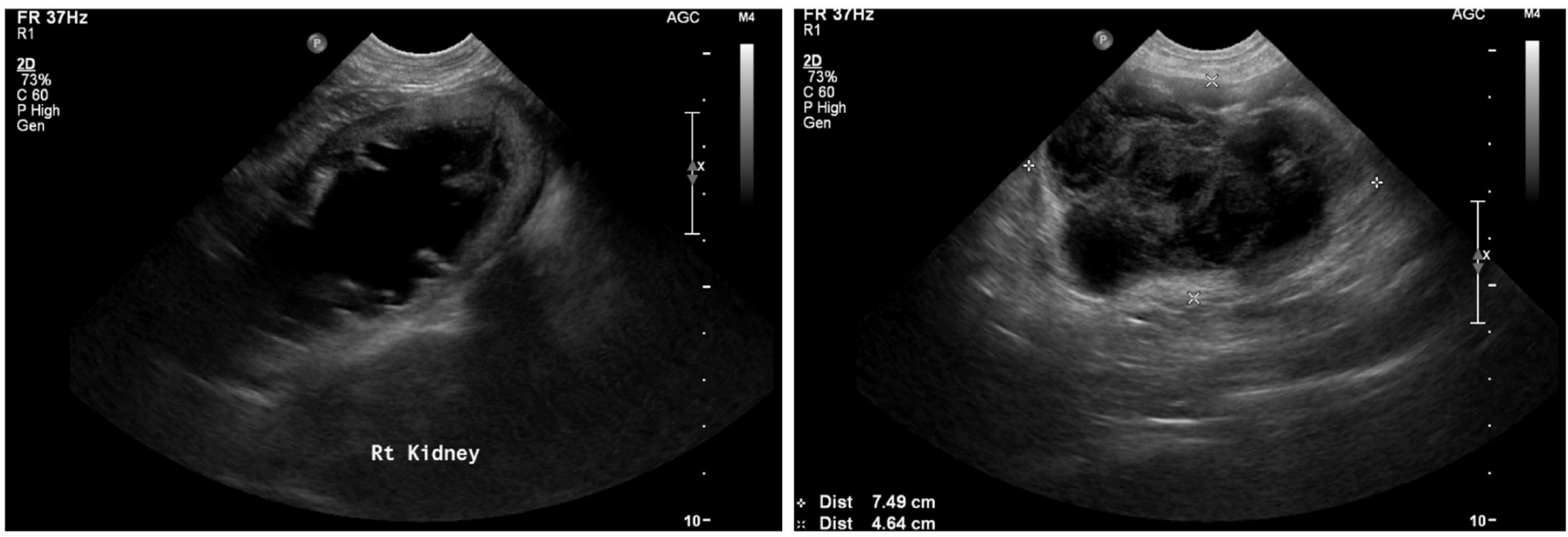

Fig. 2. (A) A focal mass $(4.6 \times 7.5 \mathrm{~cm})$ at the right ureter with $(B)$ severe hydronephrosis of the right kidney was detected. 
was left open. Following surgery, the dog's temperature was elevated $\left(39.8^{\circ} \mathrm{C}\right)$, and he was treated with cefotaxime (Cefotaxime Sodium Injection 500 mg, Shinpoong Pharm. Co.) $(20 \mathrm{mg} / \mathrm{kg}$, two or three times a day, intravenously) and aminopyrine (Aminophylline Injection (2.5\%) Daihan, Daihan Pharm. Co.) (2 ml/dose, one or two times a day, subcutaneously) for 7 days. He showed no improvement but developed anorexia, vomiting, and lethargy. We changed the antibiotics to amikacin (Amikin Injection 200 mg, Boryung Pharm. Co.) (180 mg/dose, twice a day, subcutaneously) based on the antibiotic susceptibility test. However, two days after amikacin administration, the dog showed severe lethargy and oliguria. Serum biochemical analysis revealed elevated levels of blood urea nitrogen $(56 \mathrm{mg} / \mathrm{dL}$, reference range $7-25 \mathrm{mg} / \mathrm{dL})$, creatinine (10.7 mg/dL, reference range 0.3-1.4 mg/dL), and phosphorus (10.7 mg/dL, reference range 2.9-6.6 mg/dL). In addition, a focal mass $(4.6 \times 7.5 \mathrm{~cm})$ at the right ureter, with severe hydronephrosis in the right kidney was detected using abdominal ultrasonography (Fig. 2). An acute renal failure with a ureteral mass was the suspected diagnosis; the dog was euthanatized.

Necropsy was performed, and an abnormal mass $(11 \times$ $10 \times 4.5 \mathrm{~cm}$ ) adhering to both the ureters was observed in the abdominal cavity containing approximately $10 \mathrm{~mL}$ of purple-red turbid fluid (Fig. 3). The sizes of the hydronephrotic right kidney and normal left kidney were $10 \times 5.5 \mathrm{~cm}$ and $8.5 \times 4.5 \mathrm{~cm}$, respectively. Severe atrophy of the right renal parenchyma associated with the accumulation of urine in the

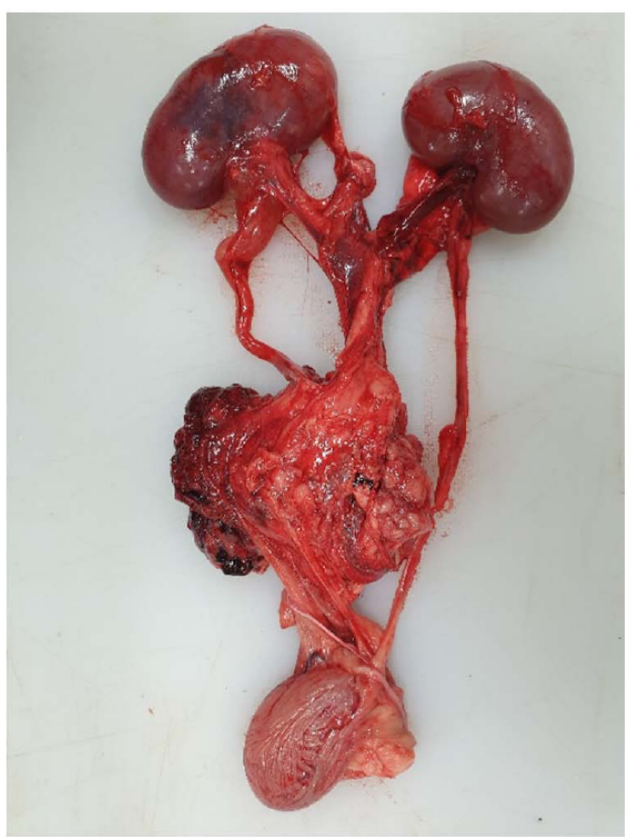

Fig. 3. Gross findings. A mass $(11 \times 10 \times 4.5 \mathrm{~cm})$ adhering to the right ureter was observed in the abdominal cavity.
A

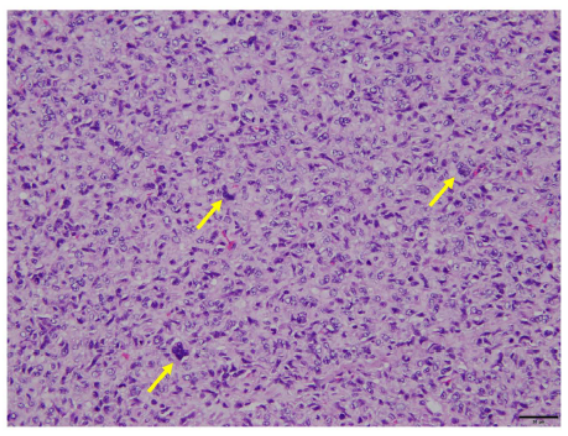

C

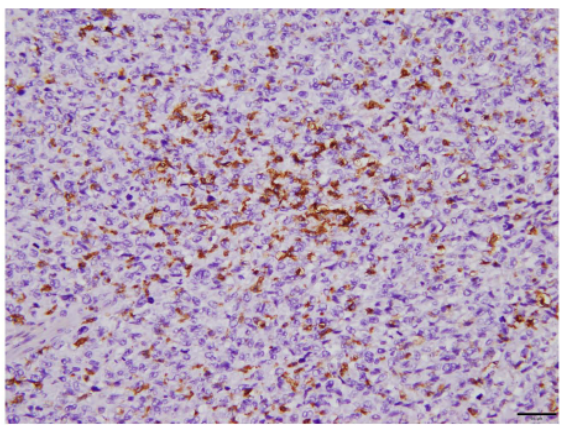

B

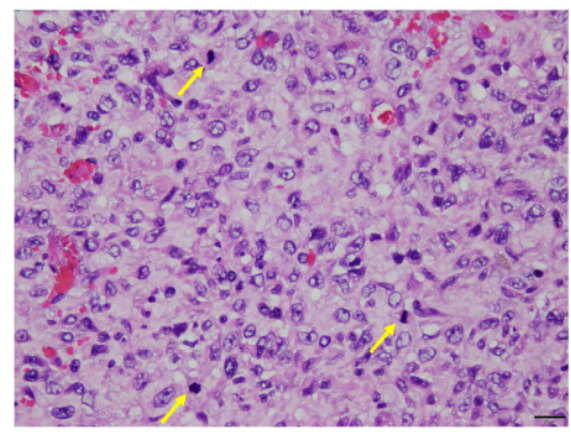

D

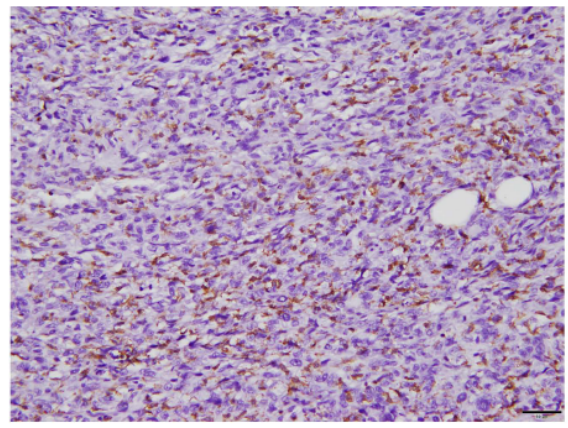

Fig. 4. Histopathologic findings. (A) The mass from skin and abdomen was composed of scattered malignant cells of a semicircular or short spindle shape and expressed (B) MHC class I, (C) Lysozyme, and (D) Iba-1. 
wide pelvis was observed on cross-sectional analysis. Compared to the left ureter, the right ureter was moderately dilated. In addition, several milky white nodules which ranged $0.5-3 \mathrm{~cm}$ in diameter were embedded in the enlarged lungs having a pale red to purple-red color. The abdominal and $\mathrm{cu}$ taneous masses of the hip were composed of many accumulated foci or scattered pleomorphic short spindle, oval cells, and multinucleated giant cells with high mitotic rate and invasive tendency to adjacent tissues (Fig. 4). These neoplastic cells expressed major histocompatibility class II, lysozyme, and Iba-1 (Fig. 4). Multifocal metastatic foci of neoplastic spindle cells and vascular thrombosis with neoplastic cell emboli were also observed in the lungs. Disseminated histiocytic sarcoma was diagnosed. In addition, hydronephrosis with a multifocal accumulation of oxalate crystals was observed in the right kidney.

\section{Discussion}

Disseminated HS is most often found in the spleen, peripheral lymph nodes, liver, bone marrow, lungs, subcutis, skeletal muscles, and kidneys $(1,3,4)$. In this case, the skin, lung, and ureter were involved. Previous studies have reported that thrombocytopenia, hypoalbuminemia, anemia, disseminated disease, and lack of antitumor treatment were negative prognostic factors $(2,9)$. In addition, Dervisis et al. (2) reported that the concurrent use of corticosteroids appeared to increase the risk of death. In this case, the disseminated form and no antitumor treatment attributed to the poor prognosis.

This dog was diagnosed with acute renal failure and was euthanized. Acute renal failure is characterized by the rapid loss of nephron function, resulting in azotemia with fluid, electrolytes, and acid-base abnormalities (6). The pathogenesis of acute renal failure is multifactorial and includes decreased intrarenal blood flow and cellular damage. Several studies have suggested potential causes of acute renal failure in dogs. These include ischemia, infarction, toxins, infectious diseases, hypercalcemia, hyperviscosity, sepsis, acute pancreatitis, and drugs (8). In this case, a ureteral obstruction by tumor and the administration of aminoglycosides are the likely causes of the acute renal failure.

A combination of negative prognostic factors (disseminated form without antitumor treatment) was observed in this case. Furthermore, the anatomical region of the tumor and the administration of aminoglycosides might have contributed to the acute renal failure. This patient was diagnosed with disseminated histiocytic sarcoma by histopathologic examination and immunohistochemistry after necropsy. Unfortu- nately, we were unable to do an ultrasound examination and skin biopsy at the first presentation. Therefore, the patient could not receive any chemotherapy which was associated with the poor prognosis (10).

\section{Conclusions}

This case is the first report of abdominal mass of DHS with ureter invasion and urinary tract obstruction resulting in acute renal failure in a Jindo dog. Several negative prognosis factors contributed to the poor prognosis for this case.

\section{Source of Funding}

This study was funded by the National Research Foundation (NRF) of Korea grant funded by the Korea government (MSIT) (No. A2021-0043).

\section{Acknowledgements}

This study was supported by the Institute for Veterinary Science, College of Veterinary Medicine, Jeju National University.

\section{Conflicts of Interest}

The authors declare no conflict of interest with respect to publication of this manuscript.

\section{References}

1. Affolter VK, Moore PF. Localized and disseminated histiocytic sarcoma of dendritic cell origin in dogs. Vet Pathol 2002; 39: 74-83.

2. Dervisis NG, Kiupel M, Qin Q, Cesario L. Clinical prognostic factors in canine histiocytic sarcoma. Vet Comp Oncol 2017; 15: 11711180.

3. Fulmer AK, Mauldin GE. Canine histiocytic neoplasia: an overview. Can Vet J 2007; 48: 1041-1043, 1046-1050.

4. Hayden DW, Waters DJ, Burke BA, Manivel JC. Disseminated malignant histiocytosis in a golden retriever: clinicopathologic, ultrastructural, and immunohistochemical findings. Vet Pathol 1993; 30: 256-264.

5. Moore PF, Affolter VK, Vernau W. Canine hemophagocytic histiocytic sarcoma: a proliferative disorder of CD11d+ macrophages. Vet Pathol 2006; 43: 632-645.

6. Mugford A, Li Rm, Humm K. Acute kidney injury in dogs and cats 1. Pathogenesis and diagnosis. InPractice 2013; 35: 253-264.

7. Rajh S, Acevedo KF, Williams G, Pargass I, Bally A, Suepaul R. A case report of disseminated canine histiocytic sarcoma in Trinidad 
and Tobago. Vet Sci 2018; 5: 9.

8. Ross L. Acute kidney injury in dogs and cats. Vet Clin North Am Small Anim Pract 2011; 41: 1-14.

9. Skorupski KA, Clifford CA, Paoloni MC, Lara-Garcia A, Barber L, Kent MS, et al. CCNU for the treatment of dogs with histiocytic sarcoma. J Vet Intern Med 2007; 21: 121-126.

10. Takahashi M, Tomiyasu H, Hotta E, Asada H, Fukushima K, Kanemoto $\mathrm{H}$, et al. Clinical characteristics and prognostic factors in dogs with histiocytic sarcomas in Japan. J Vet Med Sci 2014; 76: 661-666. 